TITLE:

\title{
Measurement of Neutrino Oscillation Parameters from Muon Neutrino Disappearance with an Off-Axis Beam
}

\section{$\operatorname{AUTHOR}(\mathrm{S}):$}

Bronner, C.; Hiraki, T.; Hirota, S.; Huang, K.; Ichikawa, A. K.; leki, K.; Ikeda, M.; ... Suzuki, K.; Takahashi, S.; T2K Collaboration

\section{CITATION:}

Bronner, C.... [et al]. Measurement of Neutrino Oscillation Parameters from Muon Neutrino Disappearance with an Off-Axis Beam. Physical Review Letters 2013, 111(21): 211803.

\section{ISSUE DATE:}

2013-11-22

URL:

http://hdl.handle.net/2433/250792

RIGHT:

(C) 2013 American Physical Society 


\section{Measurement of Neutrino Oscillation Parameters from Muon Neutrino Disappearance with an Off-Axis Beam}

K. Abe,${ }^{49}$ J. Adam,${ }^{34}$ H. Aihara, ${ }^{48,25}$ T. Akiri, ${ }^{11}$ C. Andreopoulos, ${ }^{47}$ S. Aoki ${ }^{26}$ A. Ariga, ${ }^{2}$ T. Ariga, ${ }^{2}$ S. Assylbekov, ${ }^{9}$ D. Autiero, ${ }^{31}$ M. Barbi,${ }^{41}$ G. J. Barker,${ }^{57}$ G. Barr, ${ }^{37}$ M. Bass, ${ }^{9}$ M. Batkiewicz,${ }^{15}$ F. Bay, ${ }^{13}$ S. W. Bentham, ${ }^{28}$ V. Berardi, ${ }^{20}$ B. E. Berger, ${ }^{9}$ S. Berkman, ${ }^{4}$ I. Bertram ${ }^{28}$ S. Bhadra,${ }^{61}$ F. d. M. Blaszczyk,${ }^{30}$ A. Blondel, ${ }^{14}$ C. Bojechko, ${ }^{54}$ S. Bordoni, ${ }^{17}$ S. B. Boyd ${ }^{57}$ D. Brailsford, ${ }^{19}$ A. Bravar, ${ }^{14}$ C. Bronner, ${ }^{27}$ N. Buchanan, ${ }^{9}$ R. G. Calland, ${ }^{29}$ J. Caravaca Rodríguez, ${ }^{17}$

S. L. Cartwright ${ }^{45}$ R. Castillo, ${ }^{17}$ M. G. Catanesi, ${ }^{20}$ A. Cervera, ${ }^{18}$ D. Cherdack, ${ }^{9}$ G. Christodoulou, ${ }^{29}$ A. Clifton, ${ }^{9}$ J. Coleman, ${ }^{29}$ S. J. Coleman, ${ }^{8}$ G. Collazuol, ${ }^{22}$ K. Connolly, ${ }^{58}$ L. Cremonesi, ${ }^{40}$ A. Curioni, ${ }^{13}$ A. Dabrowska, ${ }^{15}$ I. Danko, ${ }^{39}$ R. Das, ${ }^{9}$ S. Davis, ${ }^{58}$ P. de Perio, ${ }^{52}$ G. De Rosa, ${ }^{21}$ T. Dealtry,${ }^{47,37}$ S. R. Dennis,${ }^{57,47}$ C. Densham,${ }^{47}$ F. Di Lodovico, ${ }^{40}$ S. Di Luise, ${ }^{13}$ O. Drapier,${ }^{12}$ T. Duboyski,${ }^{40}$ K. Duffy,${ }^{37}$ F. Dufour, ${ }^{14}$ J. Dumarchez,${ }^{38}$ S. Dytman, ${ }^{39}$ M. Dziewiecki, ${ }^{56}$ S. Emery, ${ }^{6}$ A. Ereditato, ${ }^{2}$ L. Escudero, ${ }^{18}$ A. J. Finch,${ }^{28}$ E. Frank,${ }^{2}$ M. Friend, ${ }^{16, \dagger}$ Y. Fujii, ${ }^{16, \dagger}$ Y. Fukuda, ${ }^{32}$ A. P. Furmanski, ${ }^{57}$ V. Galymov, ${ }^{6}$ A. Gaudin, ${ }^{54}$ S. Giffin, ${ }^{41}$ C. Giganti, ${ }^{38}$ K. Gilje ${ }^{34}$ T. Golan, ${ }^{60}$ J. J. Gomez-Cadenas, ${ }^{18}$ M. Gonin, ${ }^{12}$ N. Grant,${ }^{28}$ D. Gudin, ${ }^{24}$ D. R. Hadley,${ }^{57}$ A. Haesler, ${ }^{14}$ M. D. Haigh,${ }^{57}$ P. Hamilton, ${ }^{19}$ D. Hansen, ${ }^{39}$ T. Hara, ${ }^{26}$ M. Hartz, ${ }^{25,53}$ T. Hasegawa, ${ }^{16, \dagger}$ N. C. Hastings, ${ }^{41}$ Y. Hayato, ${ }^{49}$ C. Hearty, ${ }^{4,}$ R. L. Helmer, ${ }^{53}$ M. Hierholzer, ${ }^{2}$ J. Hignight ${ }^{34}$ A. Hillairet, ${ }^{54}$ A. Himmel, ${ }^{11}$ T. Hiraki, ${ }^{27}$ S. Hirota, ${ }^{27}$ J. Holeczek, ${ }^{46}$ S. Horikawa, ${ }^{13}$ K. Huang, ${ }^{27}$ A. K. Ichikawa, ${ }^{27}$ K. Ieki, ${ }^{27}$ M. Ieva, ${ }^{17}$ M. Ikeda, ${ }^{27}$ J. Imber, ${ }^{34}$ J. Insler, ${ }^{30}$ T. J. Irvine, ${ }^{50}$ T. Ishida, ${ }^{16, \dagger}$ T. Ishii, ${ }^{16, \dagger}$ S. J. Ives, ${ }^{19}$ K. Iyogi, ${ }^{49}$

A. Izmaylov, ${ }^{18,24}$ A. Jacob, ${ }^{37}$ B. Jamieson, ${ }^{59}$ R. A. Johnson, ${ }^{8}$ J. H. Jo, ${ }^{34}$ P. Jonsson, ${ }^{19}$ K. K. Joo, ${ }^{7}$ C. K. Jung, ${ }^{34,8}$ A. C. Kaboth ${ }^{19}$ T. Kajita ${ }^{50,8}$ H. Kakuno, ${ }^{51}$ J. Kameda ${ }^{49}$ Y. Kanazawa, ${ }^{48}$ D. Karlen,${ }^{54,53}$ I. Karpikov, ${ }^{24}$ E. Kearns, ${ }^{3,8}$ M. Khabibullin, ${ }^{24}$ A. Khotjantsev, ${ }^{24}$ D. Kielczewska, ${ }^{55}$ T. Kikawa, ${ }^{27}$ A. Kilinski, ${ }^{33}$ J. Kim, ${ }^{4}$ S. B. Kim, ${ }^{44}$ J. Kisiel,${ }^{46}$ P. Kitching, ${ }^{1}$ T. Kobayashi, ${ }^{16, \dagger}$ G. Kogan, ${ }^{19}$ A. Kolaceke, ${ }^{41}$ A. Konaka, ${ }^{53}$ L. L. Kormos, ${ }^{28}$ A. Korzenev, ${ }^{14}$ K. Koseki, ${ }^{16, \dagger}$ Y. Koshio, ${ }^{35,8}$ I. Kreslo, ${ }^{2}$ W. Kropp, ${ }^{5}$ H. Kubo, ${ }^{27}$ Y. Kudenko, ${ }^{24, \|}$ S. Kumaratunga,${ }^{53}$ R. Kurjata, ${ }^{56}$ T. Kutter, ${ }^{30}$ J. Lagoda, ${ }^{33}$ K. Laihem, ${ }^{43}$ M. Laveder, ${ }^{22}$ M. Lawe,${ }^{45}$ M. Lazos,${ }^{29}$ K. P. Lee,${ }^{50}$ C. Licciardi,${ }^{41}$ I. T. Lim, ${ }^{7}$ T. Lindner, ${ }^{53}$ C. Lister, ${ }^{57}$ R. P. Litchfield, ${ }^{57}$ A. Longhin, ${ }^{22}$ G. D. Lopez, ${ }^{34}$ L. Ludovici, ${ }^{23}$ M. Macaire, ${ }^{6}$ L. Magaletti, ${ }^{20}$ K. Mahn, ${ }^{53}$ M. Malek, ${ }^{19}$ S. Manly, ${ }^{42}$ A. D. Marino, ${ }^{8}$ J. Marteau, ${ }^{31}$ J. F. Martin, ${ }^{52}$ T. Maruyama, ${ }^{16, \dagger}$ J. Marzec,${ }^{56}$ P. Masliah, ${ }^{19}$ E. L. Mathie, ${ }^{41}$ V. Matveev, ${ }^{24}$ K. Mavrokoridis, ${ }^{29}$ E. Mazzucato, ${ }^{6}$ M. McCarthy,${ }^{4}$ N. McCauley, ${ }^{29}$ K. S. McFarland, ${ }^{42}$ C. McGrew ${ }^{34}$ C. Metelko, ${ }^{29}$ P. Mijakowski, ${ }^{33}$ C. A. Miller, ${ }^{53}$ A. Minamino, ${ }^{27}$ O. Mineev, ${ }^{24}$ S. Mine,${ }^{5}$ A. Missert, ${ }^{8}$ M. Miura, ${ }^{49,8}$ L. Monfregola, ${ }^{18}$ S. Moriyama, ${ }^{49,8}$ Th. A. Mueller, ${ }^{12}$ A. Murakami, ${ }^{27}$ M. Murdoch, ${ }^{29}$ S. Murphy, ${ }^{13}$ J. Myslik, ${ }^{54}$

T. Nagasaki, ${ }^{27}$ T. Nakadaira, ${ }^{16, \dagger}$ M. Nakahata, ${ }^{49,25}$ T. Nakai, ${ }^{36}$ K. Nakamura, ${ }^{25,16, \dagger}$ S. Nakayama, ${ }^{49, \S}$ T. Nakaya, ${ }^{27,8}$ K. Nakayoshi, ${ }^{16, \dagger}$ D. Naples,${ }^{39}$ C. Nielsen, ${ }^{4}$ M. Nirkko, ${ }^{2}$ K. Nishikawa, ${ }^{16, \dagger}$ Y. Nishimura, ${ }^{50}$ H. M. O’ Keeffe,${ }^{28}$ R. Ohta, ${ }^{16, \dagger}$ K. Okumura, ${ }^{50,25}$ T. Okusawa, ${ }^{36}$ W. Oryszczak ${ }^{55}$ S. M. Oser, ${ }^{4}$ M. Otani,${ }^{27}$ R. A. Owen, ${ }^{40}$ Y. Oyama, ${ }^{16, \dagger}$ M. Y. Pac, ${ }^{10}$ V. Palladino, ${ }^{21}$ V. Paolone, ${ }^{39}$ D. Payne,${ }^{29}$ G. F. Pearce, ${ }^{47}$ O. Perevozchikov, ${ }^{30}$ J. D. Perkin,${ }^{45}$ Y. Petrov, ${ }^{4}$

E. S. Pinzon Guerra, ${ }^{61}$ C. Pistillo, ${ }^{2}$ P. Plonski,${ }^{56}$ E. Poplawska,${ }^{40}$ B. Popov, ${ }^{38, \pi}$ M. Posiadala, ${ }^{55}$ J.-M. Poutissou, ${ }^{53}$ R. Poutissou, ${ }^{53}$ P. Przewlocki, ${ }^{33}$ B. Quilain, ${ }^{12}$ E. Radicioni, ${ }^{20}$ P. N. Ratoff, ${ }^{28}$ M. Ravonel, ${ }^{14}$ M. A. M. Rayner, ${ }^{14}$ A. Redij, ${ }^{2}$ M. Reeves,${ }^{28}$ E. Reinherz-Aronis, ${ }^{9}$ F. Retiere, ${ }^{53}$ A. Robert ${ }^{38}$ P. A. Rodrigues, ${ }^{42}$ E. Rondio, ${ }^{33}$ S. Roth, ${ }^{43}$ A. Rubbia, ${ }^{13}$ D. Ruterbories, ${ }^{9}$ R. Sacco, ${ }^{40}$ K. Sakashita, ${ }^{16, \dagger}$ F. Sánchez, ${ }^{17}$ F. Sato, ${ }^{16}$ E. Scantamburlo, ${ }^{14}$ K. Scholberg, ${ }^{11,}$ J. Schwehr, ${ }^{9}$ M. Scott,${ }^{53}$ Y. Seiya ${ }^{36}$ T. Sekiguchi, ${ }^{16, \dagger}$ H. Sekiya,${ }^{49,8}$ D. Sgalaberna,${ }^{13}$ M. Shiozawa, ${ }^{49,25}$ S. Short, ${ }^{19}$ Y. Shustrov,${ }^{24}$ P. Sinclair, ${ }^{19}$ B. Smith, ${ }^{19}$ R. J. Smith, ${ }^{37}$ M. Smy, ${ }^{5}$ J. T. Sobczyk ${ }^{60}$ H. Sobel,${ }^{5,25}$ M. Sorel,${ }^{18}$ L. Southwell, ${ }^{28}$ P. Stamoulis, ${ }^{18}$ J. Steinmann, ${ }^{43}$ B. Still, ${ }^{40}$ Y. Suda, ${ }^{48}$ A. Suzuki, ${ }^{26}$ K. Suzuki, ${ }^{27}$ S. Y. Suzuki, ${ }^{16, \dagger}$ Y. Suzuki, ${ }^{49,25}$ T. Szeglowski, ${ }^{46}$

R. Tacik,${ }^{41,53}$ M. Tada,${ }^{16, \dagger}$ S. Takahashi, ${ }^{27}$ A. Takeda, ${ }^{49}$ Y. Takeuchi, ${ }^{26,25}$ H. K. Tanaka ${ }^{49,}{ }^{8}$ H. A. Tanaka, ${ }^{4, \$}$ M. M. Tanaka, ${ }^{16, \dagger}$ I. J. Taylor, ${ }^{34}$ D. Terhorst, ${ }^{43}$ R. Terri,${ }^{40}$ L. F. Thompson, ${ }^{45}$ A. Thorley,${ }^{29}$ S. Tobayama, ${ }^{4}$ W. Toki, ${ }^{9}$ T. Tomura ${ }^{49}$ Y. Totsuka, ${ }^{29, *}$ C. Touramanis,${ }^{29}$ T. Tsukamoto,${ }^{16, \dagger}$ M. Tzanov,${ }^{30}$ Y. Uchida, ${ }^{19}$ K. Ueno, ${ }^{49}$ A. Vacheret ${ }^{37}$ M. Vagins, ${ }^{25,5}$ G. Vasseur, ${ }^{6}$ T. Wachala, ${ }^{15}$ A. V. Waldron, ${ }^{37}$ C. W. Walter, ${ }^{11}{ }^{\S}$ D. Wark,${ }^{47,19}$ M. O. Wascko, ${ }^{19}$ A. Weber,${ }^{47,37}$ R. Wendell, ${ }^{49,8}$ R. J. Wilkes, ${ }^{58}$ M. J. Wilking, ${ }^{53}$ C. Wilkinson, ${ }^{45}$ Z. Williamson,${ }^{37}$ J. R. Wilson, ${ }^{40}$ R. J. Wilson, ${ }^{9}$ T. Wongjirad, ${ }^{11}$ Y. Yamada,${ }^{16, \dagger}$ K. Yamamoto, ${ }^{36}$ C. Yanagisawa, ${ }^{34, * *}$ S. Yen, ${ }^{53}$ N. Yershov, ${ }^{24}$ M. Yokoyama, ${ }^{48,8}$ T. Yuan, ${ }^{8}$ A. Zalewska, ${ }^{15}$ J. Zalipska, ${ }^{33}$ L. Zambelli, ${ }^{38}$ K. Zaremba ${ }^{56}$ M. Ziembicki, ${ }^{56}$ E. D. Zimmerman, ${ }^{8}$ M. Zito, ${ }^{6}$ and J. Żmuda ${ }^{60}$

(T2K Collaboration)

\footnotetext{
${ }^{1}$ University of Alberta, Centre for Particle Physics, Department of Physics, Edmonton, Alberta, Canada

${ }^{2}$ University of Bern, Albert Einstein Center for Fundamental Physics, Laboratory for High Energy Physics (LHEP), Bern, Switzerland
} 
${ }^{3}$ Boston University, Department of Physics, Boston, Massachusetts, USA

${ }^{4}$ University of British Columbia, Department of Physics and Astronomy, Vancouver, British Columbia, Canada

${ }^{5}$ University of California, Irvine, Department of Physics and Astronomy, Irvine, California, USA

${ }^{6}$ IRFU, CEA Saclay, Gif-sur-Yvette, France

${ }^{7}$ Chonnam National University, Institute for Universe and Elementary Particles, Gwangju, Korea

${ }^{8}$ University of Colorado at Boulder, Department of Physics, Boulder, Colorado, USA

${ }^{9}$ Colorado State University, Department of Physics, Fort Collins, Colorado, USA

${ }^{10}$ Dongshin University, Department of Physics, Naju, Korea

${ }^{11}$ Duke University, Department of Physics, Durham, North Carolina, USA

${ }^{12}$ Ecole Polytechnique, IN2P3-CNRS, Laboratoire Leprince-Ringuet, Palaiseau, France

${ }^{13}$ ETH Zurich, Institute for Particle Physics, Zurich, Switzerland

${ }^{14}$ University of Geneva, Section de Physique, DPNC, Geneva, Switzerland

${ }^{15}$ H. Niewodniczanski Institute of Nuclear Physics PAN, Cracow, Poland

${ }^{16}$ High Energy Accelerator Research Organization (KEK), Tsukuba, Ibaraki, Japan

${ }^{17}$ Institut de Fisica d'Altes Energies (IFAE), Bellaterra (Barcelona), Spain

${ }^{18}$ IFIC (CSIC \& University of Valencia), Valencia, Spain

${ }^{19}$ Imperial College London, Department of Physics, London, United Kingdom

${ }^{20}$ Dipartimento Interuniversitario di Fisica, INFN Sezione di Bari and Università e Politecnico di Bari, Bari, Italy

${ }^{21}$ Dipartimento di Fisica, INFN Sezione di Napoli and Università di Napoli, Napoli, Italy

${ }^{22}$ Dipartimento di Fisica, INFN Sezione di Padova and Università di Padova, Padova, Italy

${ }^{23}$ INFN Sezione di Roma and Università di Roma "La Sapienza”, Roma, Italy

${ }^{24}$ Institute for Nuclear Research of the Russian Academy of Sciences, Moscow, Russia

${ }^{25}$ Kavli Institute for the Physics and Mathematics of the Universe (WPI), Todai Institutes for Advanced Study, University of Tokyo, Kashiwa, Chiba, Japan

${ }^{26}$ Kobe University, Kobe, Japan

${ }^{27}$ Kyoto University, Department of Physics, Kyoto, Japan

${ }^{28}$ Lancaster University, Physics Department, Lancaster, United Kingdom

${ }^{29}$ University of Liverpool, Department of Physics, Liverpool, United Kingdom

${ }^{30}$ Louisiana State University, Department of Physics and Astronomy, Baton Rouge, Louisiana, USA

${ }^{31}$ Université de Lyon, Université Claude Bernard Lyon 1, IPN Lyon (IN2P3), Villeurbanne, France

${ }^{32}$ Miyagi University of Education, Department of Physics, Sendai, Japan

${ }^{33}$ National Centre for Nuclear Research, Warsaw, Poland

${ }^{34}$ State University of New York at Stony Brook, Department of Physics and Astronomy, Stony Brook, New York, USA

${ }^{35}$ Okayama University, Department of Physics, Okayama, Japan

${ }^{36}$ Osaka City University, Department of Physics, Osaka, Japan

${ }^{37}$ Oxford University, Department of Physics, Oxford, United Kingdom

${ }^{38}$ UPMC, Université Paris Diderot, CNRS/IN2P3, Laboratoire de Physique Nucléaire et de Hautes Energies (LPNHE), Paris, France

${ }^{39}$ University of Pittsburgh, Department of Physics and Astronomy, Pittsburgh, Pennsylvania, USA

${ }^{40}$ Queen Mary University of London, School of Physics and Astronomy, London, United Kingdom

${ }^{41}$ University of Regina, Department of Physics, Regina, Saskatchewan, Canada

${ }^{42}$ University of Rochester, Department of Physics and Astronomy, Rochester, New York, USA

${ }^{43}$ RWTH Aachen University, III. Physikalisches Institut, Aachen, Germany

${ }^{44}$ Seoul National University, Department of Physics and Astronomy, Seoul, Korea

${ }^{45}$ University of Sheffield, Department of Physics and Astronomy, Sheffield, United Kingdom

${ }^{46}$ University of Silesia, Institute of Physics, Katowice, Poland

${ }^{47}$ STFC, Rutherford Appleton Laboratory, Harwell Oxford, and Daresbury Laboratory, Warrington, United Kingdom

${ }^{48}$ University of Tokyo, Department of Physics, Tokyo, Japan

${ }^{49}$ University of Tokyo, Institute for Cosmic Ray Research, Kamioka Observatory, Kamioka, Japan

${ }^{50}$ University of Tokyo, Institute for Cosmic Ray Research, Research Center for Cosmic Neutrinos, Kashiwa, Japan

${ }^{51}$ Tokyo Metropolitan University, Department of Physics, Tokyo, Japan

${ }^{52}$ University of Toronto, Department of Physics, Toronto, Ontario, Canada

${ }^{53}$ TRIUMF, Vancouver, British Columbia, Canada

${ }^{54}$ University of Victoria, Department of Physics and Astronomy, Victoria, British Columbia, Canada

${ }^{55}$ University of Warsaw, Faculty of Physics, Warsaw, Poland

${ }^{56}$ Warsaw University of Technology, Institute of Radioelectronics, Warsaw, Poland

${ }^{57}$ University of Warwick, Department of Physics, Coventry, United Kingdom

${ }^{58}$ University of Washington, Department of Physics, Seattle, Washington, USA

${ }^{59}$ University of Winnipeg, Department of Physics, Winnipeg, Manitoba, Canada

${ }^{60}$ Wroclaw University, Faculty of Physics and Astronomy, Wroclaw, Poland

${ }^{61}$ York University, Department of Physics and Astronomy, Toronto, Ontario, Canada

(Received 31 July 2013; revised manuscript received 9 October 2013; published 19 November 2013) 
The T2K Collaboration reports a precision measurement of muon neutrino disappearance with an off-axis neutrino beam with a peak energy of $0.6 \mathrm{GeV}$. Near detector measurements are used to constrain the neutrino flux and cross section parameters. The Super-Kamiokande far detector, which is $295 \mathrm{~km}$ downstream of the neutrino production target, collected data corresponding to $3.01 \times 10^{20}$ protons on target. In the absence of neutrino oscillations, $205 \pm 17$ (syst) events are expected to be detected while only 58 muon neutrino event candidates are observed. A fit to the neutrino rate and energy spectrum, assuming three neutrino flavors and normal mass hierarchy yields a best-fit mixing angle $\sin ^{2}\left(\theta_{23}\right)=$ $0.514 \pm 0.082$ and mass splitting $\left|\Delta m_{32}^{2}\right|=2.44_{-0.15}^{+0.17} \times 10^{-3} \mathrm{eV}^{2} / c^{4}$. Our result corresponds to the maximal oscillation disappearance probability.

Introduction.-Oscillations between different neutrino flavor states are a physics process well described by the $3 \times 3$ Pontecorvo-Maki-Nakagawa-Sakata mixing matrix [1], which is parametrized [2] by three mixing angles $\theta_{12}$, $\theta_{23}, \theta_{13}$, and a $C P$ violating phase $\delta_{C P}$. In this mixing scheme, the angle $\theta_{23}$ and mass splitting $\Delta m_{32}^{2}$ are the main parameters that govern atmospheric and long-baseline $\nu_{\mu}$ disappearance oscillations. The oscillation probability in the limit $\left|\Delta m_{32}^{2}\right| \gg\left|\Delta m_{21}^{2}\right|$ is

$$
\begin{aligned}
P\left(\nu_{\mu} \rightarrow \nu_{\mu}\right) \simeq & 1-4 \cos ^{2}\left(\theta_{13}\right) \sin ^{2}\left(\theta_{23}\right)\left[1-\cos ^{2}\left(\theta_{13}\right)\right. \\
& \left.\times \sin ^{2}\left(\theta_{23}\right)\right] \sin ^{2}\left(1.27 \Delta m_{32}^{2} L / E_{\nu}\right),
\end{aligned}
$$

where $L(\mathrm{~km})$ is the neutrino propagation distance, $E_{\nu}(\mathrm{GeV})$ is the neutrino energy, and $\Delta m_{32}^{2}\left(\mathrm{eV}^{2}\right)$ is the neutrino mass splitting. Recent measurements [3-6] are consistent with the maximal $\nu_{\mu}$ disappearance for which $\theta_{23}$ is approximately $\pi / 4$. Improved knowledge of this angle has an important impact on neutrino mass models and on the interpretation of the $\nu_{e}$ appearance results, given the recent findings of nonzero $\theta_{13}$ measurements [7]. In this Letter, we report on new measurements on the values of $\sin ^{2}\left(\theta_{23}\right)$ and $\left|\Delta m_{32}^{2}\right|$.

$T 2 \mathrm{~K}$ experiment.-The $\mathrm{T} 2 \mathrm{~K}$ experiment [8] uses a $30 \mathrm{GeV}$ proton beam from the J-PARC accelerator facility. This combines (1) a muon neutrino beam line, (2) the near detector complex, which is located $280 \mathrm{~m}$ downstream of the neutrino production target and measures the neutrino beam, which constrains the neutrino flux parametrization and cross sections, and (3) the far detector, SuperKamiokande (SK), which detects neutrinos at a baseline distance of $L=295 \mathrm{~km}$ from the target. The neutrino beam is directed $2.5^{\circ}$ away from SK producing a narrowband $\nu_{\mu}$ beam [9] at the far detector whose energy peaks at $E_{\nu}=\Delta m_{32}^{2} L / 2 \pi \approx 0.6 \mathrm{GeV}$ which corresponds to the first oscillation minimum of the $\nu_{\mu}$ survival probability at SK. This enhances the sensitivity to determine $\theta_{23}$ from the oscillation measurements and reduces backgrounds from higher-energy neutrino interactions at SK.

The J-PARC main ring accelerator produces a fastextracted proton beam. The primary beam line has 21 electrostatic beam position monitors, 19 secondary emission monitors, an optical transition radiation monitor, and five current transformers which measure the proton current before a graphite target. Pions and kaons produced in the target decay in the secondary beam line, which contains three focusing horns and a 96-m-long decay tunnel. This is followed by a beam dump and a set of muon monitors.

The near detector complex contains an on-axis interactive neutrino grid detector (INGRID) [10] and an off-axis magnetic detector, ND280. A schematic detector layout is published elsewhere [8]. The INGRID detector has 14 seven-ton iron-scintillator tracker modules arranged in a $10-\mathrm{m}$ horizontal by $10-\mathrm{m}$ vertical crossed array. This detector provides high-statistics monitoring of the beam intensity, direction, profile, and stability. The off-axis detector is enclosed in a 0.2-T magnet that contains a subdetector optimized to measure $\pi^{0} \mathrm{~s}$ (PØD) [11], three time projection chambers (TPC1,2,3) [12] alternating with two one-ton fine grained detectors (FGD1,2) [13], and an electromagnetic calorimeter [14] that surrounds the TPC, FGD, and PØD detectors. A side muon range detector [15], built into slots in the magnet flux return steel, identifies muons that exit or stop in the magnet steel when the path length exceeds the energy loss range.

The SK water Cherenkov far detector [16] has a $22.5 \mathrm{kt}$ fiducial volume within a cylindrical inner detector (ID) instrumented with 11129 inward facing 20-in. phototubes. Surrounding the ID is a 2-m-wide outer detector with 1885 outward-facing 8-in. phototubes. A global positioning system with $<150 \mathrm{~ns}$ precision synchronizes the timing between SK events and the J-PARC beam spill.

These results are based on three periods: run 1 (January 2010-June 2010), run 2 (November 2010-March 2011), and run 3 (January 2012-June 2012). The proton beam power on the target steadily increased from run 1, reaching $200 \mathrm{~kW}$ with about $10^{14}$ protons per pulse on the target by the end of run 3 . The total neutrino beam exposure on the SK detector corresponds to an integrated $3.01 \times 10^{20}$ protons on target (POT).

Analysis strategy.-The analysis method estimates oscillation parameters by comparing the observed and predicted $\nu_{\mu}$ interaction rate and energy spectrum at the far detector. The rate and spectrum depend on the oscillation parameters, the incident neutrino flux, neutrino interaction cross sections, and the detector response. The initial estimate of 
the neutrino flux is determined by detailed simulations incorporating proton beam measurements, INGRID measurements, and the pion and kaon production measured by the NA61/SHINE [17] experiment. The ND280 detector measurement of $\nu_{\mu}$ charged current (CC) events constrains the initial flux estimates and parameters of the neutrino interaction models that affect the predicted rate and spectrum of neutrino interactions at both ND280 and SK. At SK, $\nu_{\mu}$ charged current quasielastic (CCQE) events are selected and efficiencies are determined, along with their dependence on final-state interactions (FSI) inside the nucleus and secondary pion interactions (SI) in the detector material. These are used in a binned likelihood ratio fit to determine the oscillation parameters.

Initial neutrino flux model.-To predict the neutrino flux at the near and far detectors, the interactions of the primary beam protons and subsequent secondary particles in a graphite target are modeled with a FLUKA2008 [18] simulation. GEANT3 [19] simulations model the secondary particles in the magnetic horns and the decay region, and their decays into neutrinos. The hadron interactions are modeled with GCALOR [20]. The simulation is tuned using measurements of the primary proton beam profile and the T2K horn magnetic fields and the NA61/SHINE hadron production results [17]. The beam direction and neutrino rate per proton on target are monitored continuously with INGRID, and the variations are less than the assigned systematic uncertainties [21]. The uncertainties in the flux are $10 \%-20 \%$ in the relevant energy range, dominated by the hadron production uncertainties. The detailed flux calculations are described elsewhere [9].

Neutrino interaction simulations and cross section parameters.-Neutrino interactions in the ND280 and SK detectors are simulated with the NEUT Monte Carlo generator [22]. External data, primarily from the MiniBooNE experiment [23], are used to tune some NEUT neutrino interaction parameters. These determine the input parameter uncertainties used in the fit to the ND280 data [21]. Neutrino interaction parameters fall into two categories: parameters that are common between ND280 and SK, and independent parameters affecting interactions at only one of the detectors. The common parameters include the axial masses for CCQE and resonant pion production, as well as five energy dependent normalizations; these are included in the fit to the ND280 data, which is discussed in the next section. Since the ND280 target is mainly carbon and differs from the SK target which is mainly oxygen, additional independent parameters are required. These affect the nuclear model for CCQE (Fermi momentum, binding energy, and spectral function modeling) and include five cross section parameters related to pion production, the neutral current (NC) cross section, the $\nu_{e} / \nu_{\mu} \mathrm{CC}$ cross section ratio, and the $\nu / \bar{\nu}$ CC cross section ratio. These independent cross section uncertainties (11 parameters) produce a $6.3 \%$ fractional error in the expected number
TABLE I. Effect of $1 \sigma$ systematic parameter variation on the number of 1-ring $\mu$-like events, computed for oscillations with $\sin ^{2}\left(\theta_{23}\right)=0.500$ and $\left|\Delta m_{32}^{2}\right|=2.40 \times 10^{-3} \mathrm{eV}^{2} / c^{4}$.

\begin{tabular}{lr}
\hline \hline Source of uncertainty (number of parameters) & $\delta n_{\mathrm{SK}}^{\exp } / n_{\mathrm{SK}}^{\exp }$ \\
\hline ND280-independent cross section (11) & $6.3 \%$ \\
Flux and ND280-common cross section (23) & $4.2 \%$ \\
Super-Kamiokande detector systematics (8) & $10.1 \%$ \\
Final-state and secondary interactions (6) & $3.5 \%$ \\
\hline Total (48) & $13.1 \%$ \\
\hline \hline
\end{tabular}

of SK events as listed in Table I. Not simulated by NEUT are multinucleon knock-out processes [24] that may affect [25] oscillation parameter determination strongly. Our estimation of the bias on the oscillation parameters from these processes appears to be smaller than the current statistical precision.

ND280 measurements, flux, and common cross section fits.-The ND280 detector measures inclusive CC events with a vertex in FGD1 located upstream of FGD2 and with the muon passing through TPC2. The event selection uses the highest-momentum negatively charged track entering TPC2 that matches a vertex inside the upstream FGD1 fiducial volume. In addition, the measured track energy loss in TPC2 must be compatible with a muon. Events originating from interactions in upstream detectors are vetoed by excluding events with a track in the TPC1 upstream of FGD1. This suppresses events with interactions occurring upstream of FGD1 or with a charged particle going backwards from FGD1 into TPC1. Using an inclusive CC selection, the efficiency is $47.6 \%$ with a purity of $88.1 \%$. The main backgrounds are events where the neutrino interactions occur outside FGD1 and migrate into the fiducial volume due to misreconstruction, or from neutral particles interacting within the FGD1.

The CC inclusive sample is further subdivided into two samples called CCQE and CCnQE. The CCQE sample is optimized to select charged current quasielastic events and the other remaining events, called CCnQE, are the charged current nonquasielastic events. This separation is made to improve constraints on the neutrino flux and cross section parameters. The CCQE selection vetoes events with additional tracks that cross FGD1 and TPC2 or have electrons from muon decay found inside FGD1. After beam and data quality cuts, there are $5841 \mathrm{CCQE}$ and $5214 \mathrm{CCnQE}$ events that correspond to an integrated data set of $2.66 \times$ $10^{20}$ POT. These two data selections are each subdivided into 5 (momentum) $\times 4$ (angular) bins which produces a 40-bin histogram used in a fit to the ND280 data.

The 40-bin histogram and cosmic-ray control samples are fit to estimate the neutrino flux crossing ND280 in 11 bins of $E_{\nu_{\mu}}$, seven common and four ND280 neutrino interaction parameters, detector response parameters, and their covariance. This ND280 fit also estimates the SK flux parameters, which are constrained through their prior 


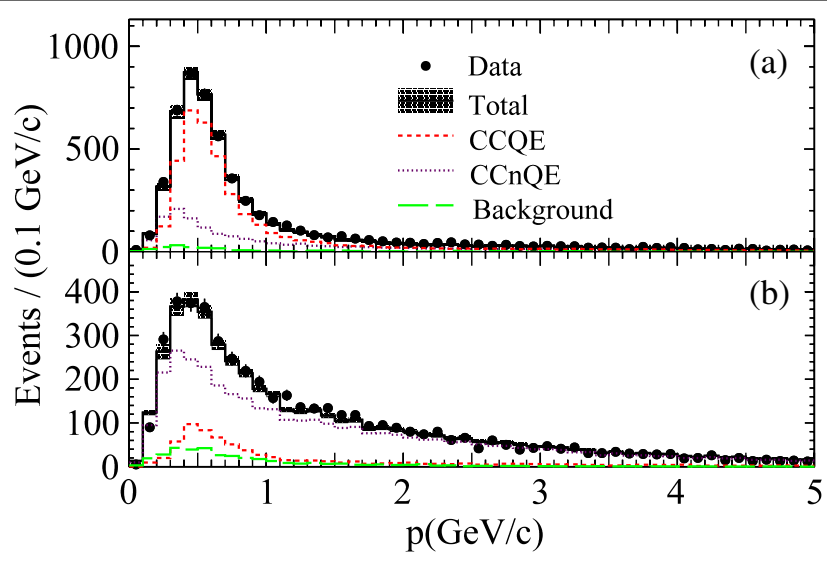

FIG. 1 (color online). The ND280 momentum data distributions of (a) the CCQE and (b) CCnQE selections. The predicted total, CCQE, CCnQE, and background event distributions from the ND280 fit are overlaid on both figures.

covariance with the ND280 flux parameters as calculated by the beam simulation described earlier. The absolute track momentum scale, pion secondary interactions, and background uncertainties are the largest detector systematics. The reconstructed ND280 $\mu^{-}$momentum distributions for CCQE and CCnQE selections and predicted event distributions from the ND280 fit to data are shown in Fig. 1. For the oscillation fits, the ND280 fit provides a systematic parameter error matrix which consists of $11 E_{\nu_{\mu}}$ SK flux normalizations, five $E_{\bar{\nu}_{\mu}}$ SK flux normalizations, and the seven common neutrino interaction parameters. The fractional error on the predicted number of SK candidate events from the uncertainties in these 23 parameters, as shown in Table I, is $4.2 \%$. Without the constraint from the ND280 measurements this fractional error would be $21.8 \%$.

SK measurements. - The SK far detector $\nu_{\mu}$ candidate events are selected from fully contained beam events. The SK phototube hits must be within $\pm 500 \mu$ s of the expected neutrino arrival time, and there must be low outer detector activity to reject the entering background. The events must satisfy visible energy $>30 \mathrm{MeV}$, exactly one reconstructed Cherenkov ring, $\mu$-like particle ID, reconstructed muon momentum $>200 \mathrm{MeV}$, and $\leq 1$ reconstructed decay electron. The reconstructed vertex must be in the fiducial volume (at least $2 \mathrm{~m}$ away from the ID walls) and "flasher" (intermittent light-emitting phototube) events are rejected. More details about the SK event selection and reconstruction are found elsewhere [16].

Assuming a quasielastic interaction with a bound neutron and neglecting the Fermi motion, the neutrino energy is deduced from the detected muon and given by

$E_{\text {reco }}=\frac{m_{p}^{2}-\left(m_{n}-E_{b}\right)^{2}-m_{\mu}^{2}+2\left(m_{n}-E_{b}\right) E_{\mu}}{2\left(m_{n}-E_{b}-E_{\mu}+p_{\mu} \cos \theta_{\mu}\right)}$,

where $p_{\mu}, E_{\mu}$, and $\theta_{\mu}$ are the reconstructed muon momentum, energy, and the angle with respect to the beam

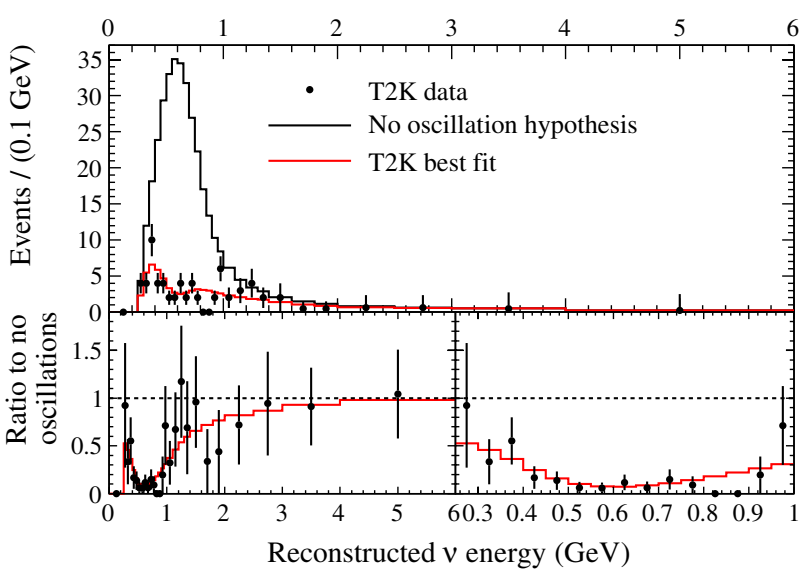

FIG. 2 (color online). The 58 event 1 -ring $\mu$-like SK reconstructed energy spectrum. Top: The two predicted curves are the no-oscillation hypothesis and the best fit from the primary oscillation analysis. The energy scale is given on the top (0-6 GeV). Bottom: The ratio of the observed spectrum over the no-oscillation hypothesis and ratio of the best-fit curve over the no-oscillation hypothesis in two energy ranges: lower left $(0-6 \mathrm{GeV})$ and lower right $(0.3-1.0 \mathrm{GeV})$. The fit uses finer binning than is shown here.

direction, respectively; $m_{p}, m_{n}$, and $m_{\mu}$ are masses of the proton, neutron, and muon, respectively, and $E_{b}=27 \mathrm{MeV}$ is the average binding energy of a nucleon in ${ }^{16} \mathrm{O}$. The $E_{\text {reco }}$ distribution of the 58 events satisfying the selection criteria is shown in Fig. 2. The no-oscillation hypothesis prediction is the solid line in Fig. 2 and the MC expectation is $205 \pm 17$ (syst) events, of which $77.7 \%$ are $\nu_{\mu}+\bar{\nu}_{\mu}$ CCQE, $20.7 \%$ are $\nu_{\mu}+\bar{\nu}_{\mu} \mathrm{CCnQE}, 1.6 \%$ are $\mathrm{NC}$, and $0.02 \%$ are $\nu_{e}+\bar{\nu}_{e} \mathrm{CC}$. The expected resolution on reconstructed energy for $\nu_{\mu}+\bar{\nu}_{\mu}$ CCQE events around the oscillation maximum is $\sim 0.1 \mathrm{GeV}$.

Eight SK detector systematic uncertainties are associated with event selection and reconstruction. The SK energy scale uncertainty is evaluated by comparing energy loss in data and $\mathrm{MC}$ calculations for samples of cosmic-ray stopping muons and associated decay electrons, as well as by comparing reconstructed invariant mass for data and MC for $\pi^{0}$ s produced by atmospheric neutrinos. The other seven SK event-selection-related uncertainties are also evaluated by comparing atmospheric neutrino $\mathrm{MC}$ and data samples. The $\nu_{\mu}+\bar{\nu}_{\mu}$ CCQE ring-counting-based selection uncertainty is evaluated in three energy bins, including correlations between energy bins. Other uncertainties result from selection criteria on the $\nu_{\mu}+\bar{\nu}_{\mu} \mathrm{CCQE}, \nu_{\mu}+\bar{\nu}_{\mu}$ $\mathrm{CCnQE}, \nu_{e}+\bar{\nu}_{e} \mathrm{CC}$, and $\mathrm{NC}$ events. These uncertainties (eight parameters) produce a $10.1 \%$ fractional error on the expected number of SK events, as listed in Table I.

Systematic uncertainties on pion interactions in the target nucleus (FSI) and SK detector (SI) are evaluated by varying underlying pion scattering cross sections in the NEUT and SK detector simulations. These uncertainties 
are evaluated separately for $\nu_{\mu}+\bar{\nu}_{\mu} \mathrm{CCQE}$ in three energy bins, $\nu_{\mu}+\bar{\nu}_{\mu} \mathrm{CCnQE}, \nu_{e}+\bar{\nu}_{e} \mathrm{CC}$, and NC events. The total FSI + SI uncertainty (six parameters) on the predicted SK event rate is $3.5 \%$ as listed in Table I.

Oscillation fits.-The oscillation parameters are estimated using a binned likelihood ratio to fit the SK spectrum in the parameter space of $\sin ^{2}\left(\theta_{23}\right),\left|\Delta m_{32}^{2}\right|$, and all 48 systematic parameters, $\boldsymbol{f}$, by minimizing

$$
\begin{aligned}
\chi^{2}\left(\sin ^{2}\left(\theta_{23}\right),\left|\Delta m_{32}^{2}\right| ; \boldsymbol{f}\right)= & \left(\boldsymbol{f}-\mathbf{f}_{\mathbf{0}}\right)^{T} \cdot \mathbf{C}^{-1} \cdot\left(\boldsymbol{f}-\mathbf{f}_{\mathbf{0}}\right) \\
& +2 \sum_{i=1}^{73} n_{i}^{\text {obs }} \ln \left(n_{i}^{\text {obs }} / n_{i}^{\text {exp }}\right) \\
& +\left(n_{i}^{\text {exp }}-n_{i}^{\text {obs }}\right) .
\end{aligned}
$$

$\mathbf{f}_{\mathbf{0}}$ is a 48-dimensional vector with the prior values of the systematics parameters, $\mathbf{C}$ is the $48 \times 48$ systematic parameter covariance matrix, $n_{i}^{\text {obs }}$ is the observed number of events in the $i$ th bin, and $n_{i}^{\exp }=n_{i}^{\exp }\left(\sin ^{2}\left(\theta_{23}\right),\left|\Delta m_{32}^{2}\right| ; \boldsymbol{f}\right)$ is the corresponding expected number of events. The sum is over 73 variable-width energy bins, with finer binning in the oscillation peak region. Oscillation probabilities are calculated using the full three neutrino oscillation framework. Normal mass hierarchy is assumed, matter effects are included with an Earth density of $\rho=2.6 \mathrm{~g} / \mathrm{cm}^{3}$ [26], and other oscillation parameters are fixed at the 2012 PDG recommended values [2] $\left[\sin ^{2}\left(2 \theta_{13}\right)=0.098, \Delta m_{21}^{2}=7.5 \times\right.$ $\left.10^{-5} \mathrm{eV}^{2} / c^{4}, \sin ^{2}\left(2 \theta_{12}\right)=0.857\right]$, and with $\delta_{C P}=0$.

The fit to the 58 events using Eq. (3) yields the best-fit point at $\sin ^{2}\left(\theta_{23}\right)=0.514 \pm 0.082$ and $\left|\Delta m_{32}^{2}\right|=$ $2.44_{-0.15}^{+0.17} \times 10^{-3} \mathrm{eV}^{2} / c^{4}$, with $\chi^{2} / n d f=56.03 / 71$. The best-fit neutrino energy spectrum is shown in Fig. 2. The point estimates of the 48 nuisance parameters are all within 0.35 standard deviations of their prior values. This fit result value combined with $\sin ^{2}\left(2 \theta_{13}\right)=0.098$ corresponds to the maximal possible oscillation disappearance probability where $\cos ^{2}\left(\theta_{13}\right) \sin ^{2}\left(\theta_{23}\right)=0.5$.

The two-dimensional confidence regions for the oscillation parameters $\sin ^{2}\left(\theta_{23}\right)$ and $\left|\Delta m_{32}^{2}\right|$ are constructed using the constant $\Delta \chi^{2}$ method [2]. The $68 \%$ and $90 \%$ contour regions are shown in Fig. 3. Also shown separately in this figure are the one-dimensional profile likelihoods for each oscillation parameter.

An alternative analysis employing a maximum likelihood fit was performed with the following likelihood function:

$$
\begin{aligned}
\mathcal{L}= & \mathcal{L}_{\text {norm }}\left(\sin ^{2}\left(\theta_{23}\right),\left|\Delta m_{32}^{2}\right|, \boldsymbol{f}\right) \\
& \times \mathcal{L}_{\text {shape }}\left(\sin ^{2}\left(\theta_{23}\right),\left|\Delta m_{32}^{2}\right|, \boldsymbol{f}\right) \mathcal{L}_{\text {syst }}(\boldsymbol{f}),
\end{aligned}
$$

where $\mathcal{L}_{\text {norm }}$ is the Poisson probability for the observed number of events, $\mathcal{L}_{\text {shape }}$ is the likelihood for the reconstructed energy spectrum, and $\mathcal{L}_{\text {syst }}$ is analogous to the first term in Eq. (3). The best-fit point is at $\sin ^{2}\left(\theta_{23}\right)=0.514$ and $\left|\Delta m_{32}^{2}\right|=2.44 \times 10^{-3} \mathrm{eV}^{2} / c^{4}$. The primary and alternative analyses are consistent; the binned maximum

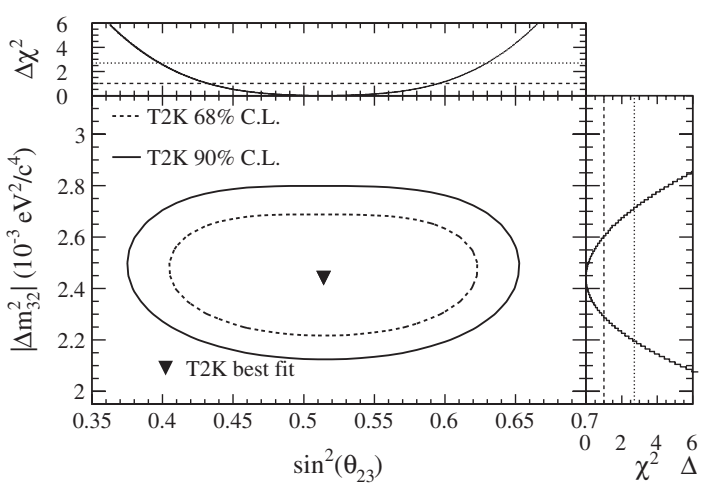

FIG. 3. The $68 \%$ and $90 \%$ C.L. contour regions for $\sin ^{2}\left(\theta_{23}\right)$ and $\left|\Delta m_{32}^{2}\right|$ are shown for the primary analysis. The onedimensional profile likelihoods for each oscillation parameter are also shown separately.

fractional difference between best-fit spectra is $1.8 \%$, and the confidence regions are almost identical.

A complementary analysis was performed, using Markov chain Monte Carlo [2] methods to produce a sample of points in the full parameter space distributed according to the posterior probability density. This analysis uses both ND280 and SK data simultaneously, rather than separately fitting the ND280 and SK measurements; the likelihood is the product of the ND280 and SK likelihoods, with the shared systematics treated jointly. The maximum probability density is found to be $\sin ^{2}\left(\theta_{23}\right)=0.516$ and $\left|\Delta m_{32}^{2}\right|=$ $2.46 \times 10^{-3} \mathrm{eV}^{2} / c^{4}$, using a uniform prior probability distribution in both $\sin ^{2}\left(\theta_{23}\right)$ and $\left|\Delta m_{32}^{2}\right|$. The contours from this analysis are similar in shape and size to the two previously described analyses, but are not expected to be identical due to the difference between Bayesian and classical intervals. This analysis also has similar results to the ND280 data fit described previously and provides a cross check.

Conclusions.-The T2K primary result (90\% C.L. region) is consistent with maximal mixing and compared to other recent experimental results in Fig. 4. In this Letter,

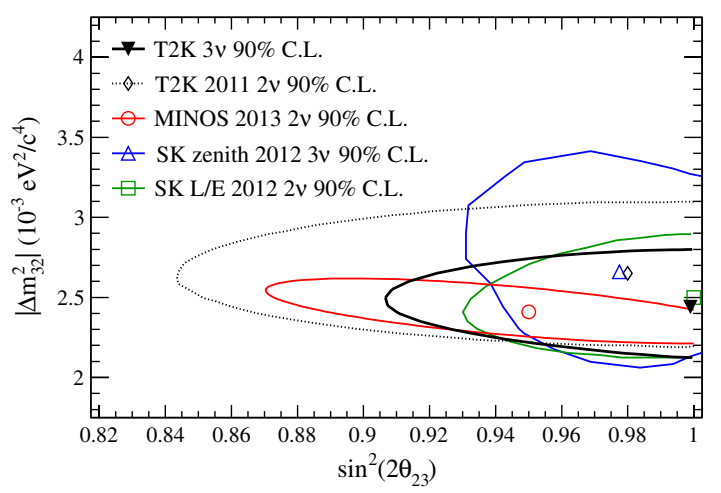

FIG. 4 (color online). The 90\% C.L. contour region for $\sin ^{2}\left(2 \theta_{23}\right)$ and $\left|\Delta m_{32}^{2}\right|$ for the primary T2K analysis is calculated by the profiling over the octant. The T2K 2011 [3], SK [27], and MINOS [6] 90\% C.L. contours with different flavor assumptions are shown for comparison. 
the $\nu_{\mu}$ disappearance analysis, based on the $3.01 \times 10^{20}$ POT off-axis beam exposure, has a best-fit mass splitting of $\left|\Delta m_{32}^{2}\right|=2.44_{-0.15}^{+0.17} \times 10^{-3} \mathrm{eV}^{2} / c^{4}$ and mixing angle, $\sin ^{2}\left(\theta_{23}\right)=0.514 \pm 0.082$. We anticipate future $\mathrm{T} 2 \mathrm{~K}$ data will improve our neutrino disappearance measurements, and our own measurements combined with other accelerator and reactor measurements will lead to important constraints and more precise determinations of the fundamental neutrino mixing parameters.

We thank the J-PARC for the superb accelerator performance and the CERN NA61 Collaboration for providing valuable particle production data. We acknowledge the support of MEXT, Japan; NSERC, NRC, and CFI, Canada; CEA and CNRS/IN2P3, France; DFG, Germany; INFN, Italy; Ministry of Science and Higher Education, Poland; RAS, RFBR, and MES, Russia; MEST and NRF, South Korea; MICINN and CPAN, Spain; SNSF and SER, Switzerland; STFC, U.K.; and DOE, U.S. We also thank CERN for the UA1/NOMAD magnet, DESY for the HERA-B magnet mover system, and NII for SINET4. In addition, the participation of individual researchers and institutions has been further supported by funds from ERC (FP7), EU; JSPS, Japan; Royal Society, U.K.; DOE Early Career program, U.S.

*Deceased.

${ }^{\dagger}$ Also at J-PARC, Tokai, Japan.

${ }^{\ddagger}$ Also at Institute of Particle Physics, Canada.

${ }^{\S}$ Also at Kavli Institute, Japan.

"Also at Moscow Institute of Physics and Technology and National Research Nuclear University, Russia.

"Also at JINR, Dubna, Russia.

**Also at BMCC/CUNY, Science Department, New York, New York, USA.

[1] B. Pontecorvo, JETP Lett. 6, 429 (1957); JETP Lett. 7, 172 (1958); JETP Lett. 26, 984 (1968); Z. Maki, M. Nakagawa, and S. Sakata, Prog. Theor. Phys. 28, 870 (1962).

[2] J. Beringer et al. (Particle Data Group), Phys. Rev. D 86, 010001 (2012); we use the standard PDG notation for the neutrino mixing angles. See Sec. 37.5 for an introduction to Markov Chains and further references. http://pdg.lbl.gov.

[3] K. Abe et al. (The T2K Collaboration), Phys. Rev. D 85, 031103 (2012).

[4] R. Wendell et al. (The Super-Kamiokande Collaboration), Phys. Rev. D 81, 092004 (2010).

[5] P. Adamson et al. (MINOS Collaboration), Phys. Rev. Lett. 108, 191801 (2012).

[6] P. Adamson et al. (MINOS Collaboration), Phys. Rev. Lett. 110, 251801 (2013).

[7] K. Abe et al. (T2K Collaboration), Phys. Rev. Lett. 107, 041801 (2011); P. Adamson et al. (MINOS Collaboration), Phys. Rev. Lett. 107, 181802 (2011); F. P. An et al.
(Daya Bay Collaboration), Phys. Rev. Lett. 108, 171803 (2012); Y. Abe et al. (Double Chooz Collaboration), Phys. Rev. Lett. 108, 131801 (2012); J. K. Ahn et al. (RENO Collaboration), Phys. Rev. Lett. 108, 191802 (2012).

[8] K. Abe et al. (T2K Collaboration), Nucl. Instrum. Methods Phys. Res., Sect. A 659, 106 (2011), see Fig. 16 for a schematic diagram of the ND280 detector.

[9] K. Abe et al. (T2K Collaboration), Phys. Rev. D 87, 012001 (2013), see the predicted flux at SK reweighted with the NA61/SHINE measurement in Fig. 39 and the neutrino events per POT as measured by the INGRID subdetector in Fig. 12.

[10] K. Abe et al. (T2K Collaboration), Nucl. Instrum. Methods Phys. Res., Sect. A 694, 211 (2012).

[11] S. Assylbekov et al. (T2K Collaboration), Nucl. Instrum. Methods Phys. Res., Sect. A 686, 48 (2012).

[12] N. Abgrall et al. (T2K Collaboration), Nucl. Instrum. Methods Phys. Res., Sect. A 637, 25 (2011).

[13] P. Amaudruz et al. (T2K Collaboration), Nucl. Instrum. Methods Phys. Res., Sect. A 696, 1 (2012).

[14] D. Allan et al., J. Instrum. 8 P10019 (2013).

[15] S. Aoki et al. (T2K Collaboration), Nucl. Instrum. Methods Phys. Res., Sect. A 698, 135 (2013).

[16] Y. Ashie et al. (Super-Kamiokande Collaboration), Phys. Rev. D 71, 112005 (2005).

[17] N. Abgrall et al. (NA61/SHINE Collaboration), Phys. Rev. C 84, 034604 (2011); 85, 035210 (2012).

[18] A. Ferrari, P. R. Sala, A. Fasso, and J. Ranft, Reports No. CERN-2005-010, No. SLAC-R-773, and No. INFNTC-05-11; G. Battistoni, F. Cerutti, A. Fassò, A. Ferrari, S. Muraro, J. Ranft, S. Roesler, and P. R. Sala, AIP Conf. Proc. 896, 31 (2007); we used FLUKA2008, which was the latest version at the time of this study.

[19] R. Brun, F. Carminati, and S. Giani, Report No. CERNW5013, 1994.

[20] C. Zeitnitz and T.A. Gabriel, in Proceedings of the International Conference on Calorimetry in High Energy Physics, 1993 (unpublished).

[21] K. Abe et al. (T2K Collaboration), Phys. Rev. D 88, 032002 (2013); see Secs. IV and V on the neutrino interaction model and the neutrino flux model.

[22] Y. Hayato, Acta Phys. Pol. B 40, 2477 (2009).

[23] A. A. Aguilar-Arevalo et al. (MiniBooNE Collaboration), Phys. Rev. D 81, 092005 (2010).

[24] J. Nieves, I. R. Simo, and M. V. Vacas, Phys. Lett. B 707, 72 (2012); M. Martini, M. Ericson, G. Chanfray, and J. Marteau, Phys. Rev. C 81, 045502 (2010).

[25] D. Meloni and M. Martini, Phys. Lett. B 716, 186 (2012); O. Lalakulich, U. Mosel, and K. Gallmeister, Phys. Rev. C 86, 054606 (2012); M. Martini, M. Ericson, and G. Chanfray, Phys. Rev. D 85, 093012 (2012); M. Martini, M. Ericson, and G. Chanfray, Phys. Rev. D 87, 013009 (2013).

[26] K. Hagiwara, N. Okamura, and K.-i. Senda, J. High Energy Phys. 09 (2011) 082.

[27] Y. Itow, Nucl. Phys. B, Proc. Suppl. 235-236, 79 (2013). 